ARTIGO

DEREVISÃO

\title{
Condicionamento físico aplicado a doenças cardiovasculares
}

\author{
Magnus Benetti ${ }^{1}$
}

\begin{abstract}
RESUMO
Tendo em vista a relevância da prevenção das doenças cardiovasculares, este artigo visa discutir alguns aspectos relativos à prevenção cardíaca primária e secundária a partir de um programa de condicionamento físico especializado. A prática da atividade física regular é utilizada tanto para a prevenção como para a reabilitação das doenças cardiovasculares. Um bom programa de condicionamento físico produz alterações fisiológicas crônicas nos sistemas cardiovascular e neurovegetativo e na secreção hormonal, constituindo expressivas modificações no que diz respeito à prevenção, estabilização e até regressão do quadro degenerativo das cardiopatias. A supervisão do paciente durante a sessão de condicionamento físico é essencial, pois a incidência de complicações cardiovasculares é maior nestes, quando comparados com adultos aparentemente saudáveis; com isso, a segurança e a qualidade do programa ficam salvaguardadas. Um aspecto muito importante é a necessidade da prescrição de exercício individualizada, tanto na prevenção primária como nas três fases de prevenção secundária. Essa prescrição deve respeitar os princípios do treinamento físico e os componentes básicos do programa: freqüência e duração, que são valores absolutos, e a intensidade, que é o fator crítico da prescrição, pois é relativa e envolve fatores como diferença entre os sexos, nível de aptidão física e idade.
\end{abstract}

\section{INTRODUÇÃO}

Estudos epidemiológicos vêm demonstrando que o índice de mortalidade por doenças do sistema circulatório tem assumido importância crescente no Brasi ${ }^{(9,12)}$. Estatística apresentada pelo Ministério da Saúde em 1988 demonstrou que as doenças do sistema circulatório eram responsáveis por $11,3 \%$ dos óbitos em 1930 e por $30 \%$ dos óbitos em $1980^{(12)}$. Já em 1984, elas eram consideradas a principal causa de morte em todas as regiões do país, exceto na região Norte ${ }^{(9,12)}$.

Estudo retrospectivo realizado no município de São Paulo demonstrou que, no período de 1940 a 1973, o índice de mortalidade por doença isquêmica do coração duplicou,

1. Professor de Fisiologia do Exercício do Centro de Educação Física e Desportos - Cefid, Coqueiros, Florianópolis, SC.

\section{Physical conditioning applied to cardiovascular diseases}

\section{ABSTRACT}

Bearing in mind the relevance of cardiovascular disease prevention, this article aims at the discussion of some relative aspects of primary and secondary cardiac prevention based on a specific physical conditioning program. The practice of regular physical activity is used both for prevention and for rehabilitation of cardiovascular diseases. A good physical conditioning program produces chronic physiological changes in the cardiovascular and neurovegetative systems, and on hormonal secretions, providing for expressive changes in the prevention, estabilization, and even regression of cardiopathic degeneration conditions. Supervision of the patient during a physical conditioning session is essential, as the incidence of cardiovascular complications is higher in these patients than in apparently healthy adults; this is a way of safeguarding the safety and quality of the program. An important aspect is the need to customize exercise prescription, both in primary prevention and in the three secondary prevention phases. This prescription should respect the principles of physical training and the basic components of the program: frequency and duration, which are absolute values, and intensity, which is the critical factor, as prescriptions involve factors such as sex, age, and physical fitness differences. 
fazendo com que essa se tornasse a maior causa de morte entre as doenças do sistema circulatório ${ }^{(8)}$. A lém disso, verificou-se que a doença coronariana foi responsável, em meados da década de 80 , por cerca de $10 \%$ do total das mortes no Brasil; esse índice atingia até $15,5 \%$ em capitais como São Paulo, Rio de J aneiro, Curitiba, Porto Alegre e Florianópolis(7).

Devido à importância que a doença coronariana vem assumindo como causa de morte, diversos estudos têm sido realizados em todo o mundo para detectar os fatores que favorecem e os que evitam seu aparecimento. Nesse sentido, o estudo de Framingham foi um dos mais expressivos, pois detectou diversos fatores de risco para a doença coronariana. Dentre esses a hipercolesterolemia, a hipertensão arterial, o tabagismo, a dislipidemia, o diabetes, a obesidade, o estresse, a taxa de fibrinogênio alta, a hiperuricemia, o hematócrito alto, a freqüência cardíaca basal alta, a capacidade vital diminuída e a vida sedentária são passíveis de controle, contrariamente aos fatores sexo, idade e história familiar ${ }^{(7)}$.

A tualmente, em diversos países do mundo, vem-se verificando diminuição na taxa de mortalidade por doenças cardiovasculares. Essa tendência também tem sido observada no município de São Paulo desde 1976. Esse declínio é atribuído às melhorias no tratamento médico e à ampliação da prevenção primária, a qual visa diminuir os fatores de risco da população(7).

Em vista da relevância da prevenção das doenças cardiovasculares, este texto pretende discutir alguns aspectos relativos à prevenção cardíaca primária e secundária a partir de um programa de condicionamento físico especializado.

\section{PREVENÇÃO PRIMÁRIA E SECUNDÁRIA DA DOENÇA CARDIOVASCULAR}

Diversos estudos têm evidenciado associação entre a inatividade e o desenvolvimento de doenças cardiovasculares; a mais importante é, sem dúvida, a doença coronariana. Outros estudos sugerem que a atividade física previne as manifestações clínicas dessa doença(14).

Dessa maneira, a atividade física pode ser utilizada tanto na prevenção do aparecimento das doenças cardiovasculares em indivíduos que têm um ou mais fatores de risco prevenção primária(14,16) - como também na prevenção das manifestações clínicas dessas doenças em pacientes cardiopatas - prevenção secundária(2).

A reabilitação é o processo pelo qual a pessoa retorna a estado ótimo físico, social, emocional, psicológico e ocupacional após algum evento patológico cardiovascular. A té a década de 60-70, o exercício, como conduta terapêutica, era pouco utilizado no processo de reabilitação(2). Contudo, a partir das últimas duas décadas, o condicionamento físico tem sido amplamente utilizado e hoje é o ponto central de

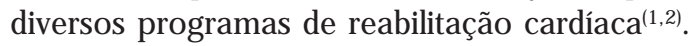

\section{BENEFÍCIOS DE UM PROGRAMA DE CONDICIONAMENTO FÍSICO}

O condicionamento físico adequado produz alterações no funcionamento cardiovascular, na secreção hormonal, nos fatores de risco das doenças cardiovasculares e sobre os fatores psicológicos. Todas essas modificações contribuem de maneira expressiva para a prevenção das doenças cardiovasculares.

\section{Efeitos sobre o sistema cardiovascular}

A maioria dos estudos demonstra que o exercício físico adequado aumenta a capacidade aeróbia máxima. Segundo Greenland e Chu(6), após infarto do miocárdio, tanto os pacientes condicionados como os não condicionados atingem capacidade máxima de nove METs, a qual é suficiente para a retomada das atividades diárias. Os condicionados, porém, podem ter consumo máximo de oxigênio $\mathrm{NO}_{2}$ máx.) até $25 \%$ maior que os não condicionados. Embora o aumento da capacidade aeróbia máxima nem sempre seja acompanhado de melhora clínica, parece inquestionável que esse aumento tem alta correlação com a qualidade de vida dos pacientes. Outro aspecto de interesse quanto ao efeito do condicionamento físico é o grau inicial de capacidade física dos pacientes, pois os mais beneficiados pelo condicionamento físico são aqueles que têm menor capacidade inicial. Isso evidencia ainda mais a importância do condicionamento físico na reabilitação cardíaca(6).

Q uanto aos principais efeitos do condicionamento físico no sistema cardiovascular, pode-se dizer que ele aumenta o volume sistólico máximo, o débito cardíaco máximo, o volume diastólico final e a massa ventricular e diminui a freqüência cardíaca de repouso e de exercício submáximo, a pressão arterial de indivíduos hipertensos e o produto da pressão arterial pela freqüência cardíaca para uma mesma carga ${ }^{(5,14)}$. Entretanto, sua ação sobre a contratilidade cardíaca, a fração de ejeção e a perfusão miocárdica é questionável(5,6). Em nível periférico, o condicionamento físico provoca aumento da densidade capilar nas fibras musculares e eleva a atividade específica de enzimas oxidativas, aumentando a diferença arteriovenosa de oxigênio(14). Em pacientes coronariopatas, o efeito do condicionamento físico parece provocar mais modificações periféricas do que modificações centrais ${ }^{(3,15)}$.

\section{Efeitos sobre a secreção hormonal}

Q uanto ao sistema hormonal, o condicionamento físico diminui a secreção de insulina, a qual é reconhecidamente aterogênica, e diminui a secreção de catecolaminas ${ }^{(14)}$. A menor concentração de catecolaminas reduz as anormalidades eletrocardiográficas, diminuindo também as arritmias ${ }^{(14)}$. A diminuição da freqüência cardíaca de repouso e de exercício submáximo aumenta o tempo de diástole, o que pode permitir maior perfusão coronariana e diminuição do consumo de oxigênio do miocárdio para uma mesma carga 
absoluta $^{(14)}$. Esses mecanismos de adaptação aumentam a tolerância ao esforço e, conseqüentemente, o limiar de angina ou de depressão de segmento $\mathrm{ST}^{(5)}$.

\section{Efeito sobre os fatores de risco}

O condicionamento físico tem ações comprovadas sobre diversos fatores de risco ${ }^{(6)}$. Os exercícios físicos regulares diminuem 0 peso, a pressão arterial|(5,14) e a concentração plasmática de triglicérides ${ }^{(5,11,14)}$, colesterol-LDL e colesterol-VLDL, e aumentam os níveis plasmáticos de colesterol$\operatorname{HDL}(4,5,11,13,14,17)$.

\section{Efeito sobre os fatores psicológicos}

Freqüentemente, pacientes com problemas cardíacos apresentam problemas psicológicos, ocupacionais e sociais(6). Embora alguns estudos nesta área apresentem limitações metodológicas, é possível sugerir que o condicionamento físico melhora o bem-estar, a autoconfiança, a depressão, a hipocondria, a tensão, a fadiga, o sono e a libido ${ }^{(5,6)}$.

\section{SEGURANÇA NOS PROGRAMAS DE REABILITAÇÃO}

A incidência de complicações cardiovasculares é maior entre pacientes cardiopatas do que entre adultos saudáveis. O risco de parada cardíaca durante o exercício físico é maior nos exercícios intensos e logo após sua finalização. Isso se dá porque durante o exercício intenso há aumento do consumo de oxigênio do miocárdio e encurtamento da diástole, o que diminui o tempo de perfusão coronariana, provocando um déficit temporário de oxigênio no tecido subendotelial. Esse déficit é ainda exacerbado pela diminuição do retorno venoso imediatamente após o término do exercício. Essa isquemia temporária pode alterar a despolarização e a velocidade de condução, levando a arritmias que, em casos extremos, podem evoluir para taquicardia ventricular ou fibrilação. Outros fatores do exercício como o desequilíbrio sódio/potássio e o excesso de catecolaminas circulantes são também agentes arritmogênicos ${ }^{(5)}$. A pesar desses aspectos, Van Camp e Peterson, apud Greenland e $\mathrm{Chu}^{(6)}$, verificaram em dados estatísticos de 167 programas de reabilitação cardíaca, somando um total de 51.303 pacientes, que o índice de parada cardíaca era de 1 por 111.996 pacientes/ hora, o índice do infarto do miocárdio não fatal era de 1 por 293.990 pacientes/ hora e o índice de morte súbita era de 1 por 783.972 pacientes/ hora. A partir desses resultados os autores concluíram que a prática corrente de reabilitação cardíaca permite prescrever exercícios supervisionados a pacientes com doenças cardiovasculares com baixo risco de complicações. Outro dado estatístico interessante é de que as paradas cardíacas parecem ocorrer mais freqüentemente em pacientes que excedem seus limites de freqüência cardíaca ou que apresentam alterações eletrocardiográficas consideradas importantes ${ }^{(6)}$. Portanto, a supervisão do paciente durante a sessão de condicionamento físico e a precisa ca- racterização de seu estado clínico e funcional são essenciais para o êxito e a segurança do programa de condicionamento físico.

\section{RECOMENDAÇÕES DE PRESCRIÇÃO DE ATIVIDADE FÍSICA}

A prescrição de um programa de condicionamento físico deve prever a freqüência, a duração, a intensidade, o tipo de exercício e a melhor forma de monitorização para que os efeitos benéficos sejam alcançados e os riscos de complicações, diminuídos ${ }^{(1)}$.

\section{Prevenção primária}

Para obter os benefícios cardiovasculares anteriormente mencionados são recomendados exercícios rítmicos aeróbios que envolvam grandes grupos musculares, como correr, nadar, andar de bicicleta, etc. M elhoras na capacidade cardiorrespiratória são obtidas com treinamentos em intensidades que variam entre 50 e $85 \%$ de $\mathrm{VO}_{2}$ máx., ou seja, 65 a $90 \%$ da freqüência cardíaca máxima ( $F C$ máx.), com duração de 15 a 60 minutos e com freqüência de três a cinco sessões por semana. Para indivíduos iniciantes recomenda-se utilizar intensidades entre 40 e $60 \%$ da capacidade funcional, evoluindo-se para intensidades entre 60 e $70 \%$ (1).

A intensidade desses exercícios é prescrita para cada indivíduo em função de sua capacidade funcional, a qual é aferida por teste ergométrico realizado antes de iniciar 0 programa de condicionamento físico. A intensidade de treinamento (freqüência cardíaca que deve ser mantida durante as sessões de condicionamento físico - FCtr) para indivíduos iniciantes sedentários é estabelecida entre 50 e $70 \%$ da freqüência cardíaca de reserva (FCres), o que é calculado da seguinte forma:

FCtr mínima $=(F C$ máx $-F C$ rep $) \times 50 \%+F C$ rep

$\mathrm{FC}$ tr máxima $=(\mathrm{FC}$ máx $-\mathrm{FC}$ rep) $\times 70 \%+\mathrm{FC}$ rep, em que $F C$ máx é a freqüência cardíaca máxima obtida no teste ergométrico e FCrep é a freqüência cardíaca medida durante o eletrocardiograma de repouso. Para indivíduos condicionados, essa freqüência de treinamento é estabelecida entre 60 e $80 \%$ da FCres. Nesse programa a freqüência de sessões é de três por semana.

Como as melhoras mais expressivas na condição física normamente ocorrem nas primeiras seis a oito sessões de exercício, é importante que após duas semanas a carga total de trabalho por sessão seja aumentada progressivamente, através de incremento na intensidade, na duração ou em ambas $^{(1)}$. No programa de condicionamento físico, essa progressão de carga é feita semanalmente por avaliação da freqüência cardíaca e pressão arterial atingida nas sessões de treinamento e através de avaliações ergométricas realizadas aos três e seis meses do programa e, a partir de então, de seis em seis meses. 


\section{Prevenção secundária}

O programa de condicionamento para a prevenção secundária pode ser dividido em três fases. Esse programa é normalmente oferecido a pacientes com doença arteriosclerótica e com deficiência da função miocárdica e da capacidade funcional, os quais podem apresentar ou não sintomas, sinais e manifestações clínicas da doença(1).

\section{Programa de condicionamento - fase I}

Esse tipo de programa é oferecido a internados no hospital, normalmente pacientes na fase pós-infarto ou pós-operatório. Essa fase do condicionamento visa fazer com que os pacientes retornem a suas tarefas diárias, recuperando os danos na capacidade funcional provocados pela doença e pela inatividade prolongada. Para esse tipo de programa faz-se necessário que cada paciente seja acompanhado por profissional especializado e que haja monitorização eletrocardiográfica durante as sessões, além de uma equipe de emergência pronta para atuar(1).

A A merican College of Sports M edicine ${ }^{(1)}$ recomenda que: na fase inicial (um a três dias pós-cirurgia ou infarto) sejam ministradas atividades de baixa intensidade (um a três METs) com gasto energético equivalente ao das atividades de autocuidado. Essas atividades devem progredir da posição deitada para a posição sentada e, em seguida, para a posição em pé. Entre três e cinco dias pós-evento, a caminhada em esteira ou os exercícios com cicloergômetro já podem ser iniciados. Nesse momento recomendam-se duas a quatro sessões diárias de exercícios, com duração de cinco a dez minutos, em intensidade de 40 a $60 \%$ do $\mathrm{VO}_{2}$ máx, o qual deve ser estimado entre três e cinco METs. Muitas vezes, utiliza-se para o condicionamento físico freqüência de 20 a 30 batimentos acima da freqüência cardíaca de repouso. Caso haja dificuldade em manter a duração do exercício, deve-se aumentar a freqüência de sessões por dia. Mas, à medida que o tempo passa, a duração deverá ser aumentada para 20 a 30 minutos e a freqüência será então diminuída para uma ou duas sessões por dia.

\section{Programa de condicionamento - fase II}

Esse programa é uma continuação da fase I e tem seu início após a alta hospitalar. A relação entre pacientes e professores de educação física deve variar entre 1:1 e 1:5 e o local onde se realiza o programa precisa ter equipamento de emergência, supervisão médica e condições para se fazer monitorizações eletrocardiográficas ${ }^{(1)}$.

$\mathrm{Na}$ fase II do programa de condicionamento físico atendem-se basicamente pacientes pós-operatório, pós-infarto do miocárdio e pós-angioplastia. Nessa fase, nosso objetivo é aumentar gradativamente a resistência dos pacientes a fim de possibilitar que eles retornem ao trabalho e a suas atividades físicas diárias. Esse programa tem duração de dois meses e é executado após teste ergométrico de protocolo de baixa intensidade. A intensidade do exercício aeróbio depende da capacidade física do paciente, atingindo até 7,5 METs. Durante esse perío do de dois meses os pacientes são reavaliados aos 30 e 60 dias. Segundo a A merican College of Sports M edicine ${ }^{(1)}$ esse programa deve encerrar-se quando os pacientes tiverem atingido capacidade funcional acima de cinco METs, estado médico estável, capacidade física (força e resistência muscular) adequada às tarefas diárias, souberem manter sua prescrição na faixa desejável e souberem reconhecer sinais e sintomas de intolerância ao exercício.

\section{Programa de condicionamento - fase III}

Essa fase é oferecida tanto a pacientes que passaram pelas fases I e II, como àqueles que não passaram por essas fases. 0 programa normalmente inclui pacientes em fase de seis a 12 meses pós-alta hospitalar. Nesse programa são aceitos pacientes com angina estável ou decrescente, arritmias controladas durante o exercício, com conhecimento de seus sintomas e com hipertensão controlada. A relação professor de educação física e pacientes não deve exceder 1:10 e deve haver rotina de pronto-atendimento que inclua equipamentos e pessoas devidamente treinadas para atender a intercorrências. Desde que necessária, a monitorização eletrocardiográfica deve ser utilizada(1).

$\mathrm{Na}$ fase III do programa de condicionamento físico, os pacientes participam das sessões como na prevenção primária e têm sua intensidade de treinamento físico estabelecida entre 50 e $70 \%$ da FCres. No entanto, para os indivíduos que apresentam isquemia ou angina durante 0 teste ergométrico, a frequêencia cardíaca máxima utilizada para calcular a freqüência de treinamento físico é aquela na qual a isquemia começou durante 0 teste ergométrico(2).

\section{CONSIDERAÇÕES ESPECIAIS}

\section{Pacientes com angina}

A American College of Sports Medicine recomenda que pacientes com angina tenham suas respostas isquêmicas avaliadas antes, durante e após o exercício. Porém, como em muitos pacientes o início da angina é observado em níveis baixos de exercício (dois a três METs), faz-se necessário o emprego de protocolos de teste ergométrico que iniciem com cargas baixas ( 1,5 a dois METs) e que tenham incrementos pequenos $(0,5 \mathrm{MET})$, a fim de facilitar a identificação do limiar de angina e das melhoras obtidas com o condicionamento físico(2).

\section{Medicamentos}

Muitos dos medicamentos utilizados por pacientes cardiopatas modificam as respostas cardiovasculares e metabólicas ao exercício. Portanto, ao prescrever exercícios para esses pacientes, deve-se levar em conta o efeito desses medicamentos. Para tanto, aconselha-se que a prescrição de exercícios seja feita baseada nas respostas obtidas em teste 
ergométrico feito sob a vigência dos medicamentos que o paciente costuma utilizar durante as sessões de treinamento ${ }^{(1)}$.

\section{CONCLUSÃO}

Baseado nos dados bibliográficos apresentados anteriormente e na experiência obtida no programa de condicionamento físico para prevenção cardiológica primária e secundária (fases II e III), pode-se dizer que programas de condicionamento físico devidamente planejados e executados, respeitando o princípio da individualidade, são benéficos para pacientes com doenças do sistema cardiovascular.

\section{REFERÊNCIAS}

1. A merican College of Sports M edicine: Guidelines for exercise testing and prescription, Philadelphia, Lea \& Febiger, 1996.

2. Berra K: Cardiac and pulmonary rehabilitation: historical perspectives and future needs. J Cardiop Rehab 11: 8-15, 1991.

3. Boykin J C, H edback B, Durstine J L: Central or peripheral adaptations during a twelve week exercise program for cardiac patients. J Cardiop Rehab 11: 311, 1991.

4. Brown D, Loy SF, Hall SJ, H eng M, Shaw S, H olland GJ : Effects of 10 weeks of work equivalent high vs. low intensity training of middle aged males aerobic capacity. J Cardiop Rehab 11: 320, 1991.

5. Franklin BA, Gordon S, Timmis G: A mount of exercise necessary for the patients with coronary artery disease. AmJ Cardiol 69: 1426-1432, 1992.

6. Greenland P, Chu J S: Efficacy of cardiac rehabilitation services with emphasis on patients after myocardial infarction. Ann Intern Med 15: 650663, 1988.
7. Laurenti R, Azevedo AC: Aspectos epidemiológicos e custo econômico-social da isquemia miocárdica, 8 a j ornada Científica do Pró-Cardíaco. "Simpósio sobre Prevenção em Cardiologia, A tividade Física e M eioAmbiente", 27-30, Rio de J aneiro, 1992.

8. Laurenti R, Fonseca LAM: A evolução da mortalidade por doença isquêmica do coração no município de São Paulo de 1940 a 1973. Arq Bras Cardiol 30: 351-355, 1977.

9. Lessa I: As doenças cerebrovasculares e as isquêmicas do coração como causa de morte no Brasil. Medicina Prat-K 4: 65-68, 1990.

10. Milani RV, Lavie CJ : Factors predicting reduction in triglycerides values following cardiac rehabilitation exercise program. J Cardiop Rehab 11: 304, 1991.

11. Milani RV, Lavie CJ : Factors predicting improvement in high-density lipoprotein cholesterol with cardiac rehabilitation and exercise programs. J Cardiop Rehab 11: 302, 1991.

12. Ministério da Saúde: Doenças crônico-degenerativas: evolução e tendências atuais - I, Centro de Documentação do Ministério da Saúde, Brasília, 1988.

13. Murray PM, Herrington DM, Davis S, Miller HS: Modest physical training in cardiac rehabilitation program associated with significant elevations in high density lipoprotein. J Cardiop Rehab 11: 303, 1991.

14. O berman A: Exercise and the primary prevention of cardiovascular disease. Am J Cardiol 55: 10D-20D, 1985

15. Rousseau MF, Brasseur LA, Detry J -MR: Hemodynamic determinants of maximal oxygen intake in patients with healted myocardial infarction: influence of physical training. Circulation 48: 943-949, 1973.

16. Squires RW: Primary prevention of coronary artery disease: the cardiovascular health clinic approach, American Association of Cardiovascular and Pulmonary Rehabilitation, Sixth Annual Meeting, 1991, p. 71-81.

17. Zema MJ , A guetti J , O gilvie PH: Exercise training in patients with isolated low HDL cholesterol. J Cardiop Rehab 11: 304, 1991. 